\title{
REGULAR BOUNDARY CONDITIONS FOR ORDINARY DIFFERENTIAL OPERATORS
}

\author{
BY \\ STEPHEN SALAFF
}

Introduction. The Birkhoff theory for linear differential operators of order $m$ treats operators determined by a nonselfadjoint differential expression and twopoint boundary conditions. In his classic of 1908 [1], Birkhoff gave determinantal inequalities, involving the leading coefficients in the boundary forms, from which asymptotic eigenfunction estimates and an expansion theorem follow. Stone [14] then showed that the Birkhoff expansion is in a sense equivalent to the Fourier expansion.

In the present paper we focus on a question of Kamke [5, p. 197], and show that if $m$ is even, selfadjoint boundary conditions meet the determinantal inequalities (which are somewhat simplified in the process): This result is not unexpected, for if the differential expression is formally selfadjoint, the operator determined is selfadjoint and an expansion theorem (such as the Fourier expansion) is available.

The difficulties in obtaining the result are (linear) algebraic ones. Consider the matrix $\left(f\left(x_{j}+x_{k}\right)\right)_{j, k=1}^{n}$, where $x_{1}<x_{2}<\cdots<x_{n}$. Let $f$ satisfy the functional equation $f(x+y)=g(x) g(y)[h(x)+h(y)]^{-1}$, where $g$ and $h$ are suitably restricted. In Lemma III we use the function $f(x)=\operatorname{cosec} x$ to show that the given matrix, and a class of others related to its compounds, are positive definite. This development is central to the proof of our theorem.

\section{Preliminaries.}

1. Notation. In this section $C^{2 m}$ denotes the $2 m$ dimensional complex vector space, with inner product $(x, y)=\sum_{j=1}^{2 m} x_{j} \bar{y}_{j}, x=\left(x_{1}, \ldots, x_{2 m}\right), y=\left(y_{1}, \ldots, y_{2 m}\right)$. The Hilbert space $H=L^{2}(0,1)$ is the space of Lebesgue square-integrable functions in the closed unit interval, with inner product

$$
(u, v)=\int_{0}^{1} u(t) \overline{v(t)} d t
$$

The subset $H_{1}$ of $H$ consists of those functions having absolutely continuous derivatives to order $m-1$, with $m$ th derivative in $H$. For $u \in H_{1}$ we define $\hat{u} \in C^{2 m}$ by $\hat{u}=\left(u(0), u^{\prime}(0), \ldots, u^{(m-1)}(0), u(1), \ldots, u^{(m-1)}(1)\right)$, where $u^{(j)}$ is the $j$ th derivative of $u$.

The transposed complex conjugate of the matrix $B$ is written $B^{*}$. The large cross $X$ which occurs in matrices and equations of this chapter will indicate the presence of certain terms whose value is not of present concern.

Received by the editors February 15, 1967. 
2. The differential operator $L$. The linear differential expression $l$, of arbitrary order $m$, is defined in $H_{1}$ by

$$
l u=\sum_{j=0}^{m} p_{m-j} u^{(j)},
$$

where $p_{0} \equiv 1$ and each $p_{j}$, together with its derivatives to order $m-j$, is in $H$. The formal adjoint expression $l^{*}$ is given for $v \in H_{1}$ by

$$
l^{*} v=\sum_{j=0}^{m}(-1)^{j}\left(\bar{p}_{m-j} v\right)^{(j)} .
$$

Lagrange's formula [2, p. 285] is

$$
(l u, v)-\left(u, l^{*} v\right)=(A \hat{u}, \hat{v})
$$

Here $A$ is a nonsingular $2 m \times 2 m$ matrix of the form

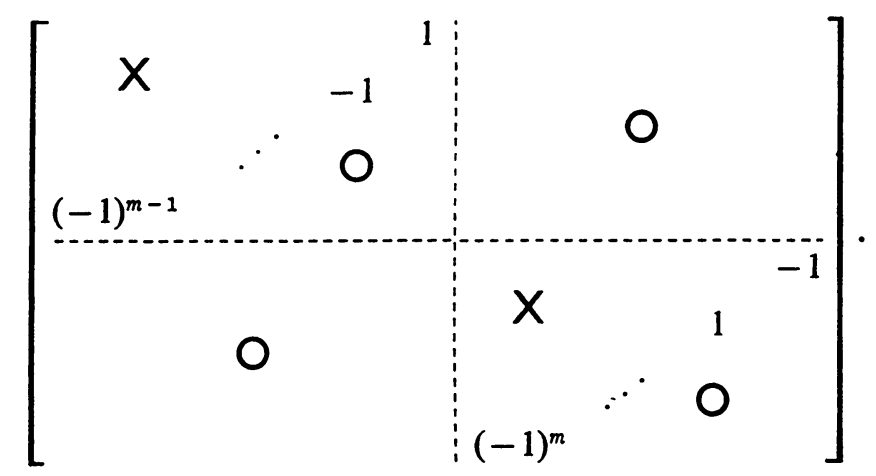

There will be $m$ linearly independent, homogeneous, two-point boundary conditions

$$
b u \equiv \sum_{j=0}^{m-1} B_{j}^{0} u^{(j)}(0)+B_{j}^{1} u^{(j)}(1)=0 .
$$

The complex column vectors $B_{j}^{0}, B_{j}^{1}$ are of length $m$. Independence requires that rank $B=m$, where $B$ is the $m \times 2 m$ matrix

$$
\left(B_{0}^{0}, \ldots, B_{m-1}^{0}, B_{0}^{1}, \ldots, B_{m-1}^{1}\right) \text {. }
$$

Thus $b u=0$ can also be written $B \hat{u}=0$. We shall refer to the left side of a boundary condition as a boundary form. Such a form is said to be of order $k, 0 \leqq k \leqq m-1$, if the highest order derivative of $u$ appearing in it (at either endpoint 0 or 1 ) is the $k$ th derivative.

Any boundary conditions obtained from $B \hat{u}=0$ by taking linear combinations (left-multiplying $B$ by a nonsingular $m \times m$ matrix $T$ ) are termed 'equivalent' conditions, since $B \hat{u}=0$ if and only if $T B \hat{u}=0$. We choose a particular $T$, corresponding to the 'normalized' boundary forms, which will now be described. 
Let $r_{m-1}=\operatorname{rank}\left(B_{m-1}^{0}, B_{m-1}^{1}\right)$. Then $r_{m-1}$ is the number $(0,1$ or 2$)$ of independent boundary forms of order $m-1$. There is a nonsingular $m \times m$ matrix $T_{m-1}$, and there are two short column vectors $b_{m-1}^{0}, b_{m-1}^{1}$, of length $r_{m-1}$, such that

$$
T_{m-1}\left(B_{m-1}^{0}, B_{m-1}^{1}\right)=\left[\begin{array}{ll}
0 & 0 \\
\vdots & \vdots \\
0 & 0 \\
b_{m-1}^{0} & b_{m-1}^{1}
\end{array}\right] .
$$

If $r_{m-1}=0$, this matrix consists of zeros only, $T_{m-1}$ can be taken as the identity, and the columns $b_{m-1}^{0}, b_{m-1}^{1}$ will be empty. Now cross off the last $r_{m-1}$ rows of $T_{m-1}\left(B_{m-2}^{0}, B_{m-2}^{1}\right)$, and let $r_{m-2}$ be the rank of the matrix remaining. Again, $r_{m-2}$ is 0,1 or 2 , and there are $T_{m-2}, b_{m-2}^{0}, b_{m-2}^{1}$ such that

$$
T_{m-2} T_{m-1}\left(B_{m-2}^{0}, B_{m-2}^{1}\right)=\left[\begin{array}{ll}
0 & 0 \\
\vdots & \vdots \\
0 & 0 \\
b_{m-2}^{0} & b_{m-2}^{1} \\
\mathrm{X} & \mathrm{X}
\end{array}\right] .
$$

The crosses indicate the presence of $r_{m-1}$ rows.

Continuing in this way till no further forms remain, we obtain the normalized boundary forms, with $T=T_{0} \cdots T_{m-2} T_{m-1}$. The normalized boundary matrix, hereafter referred to as $B$, will have the form

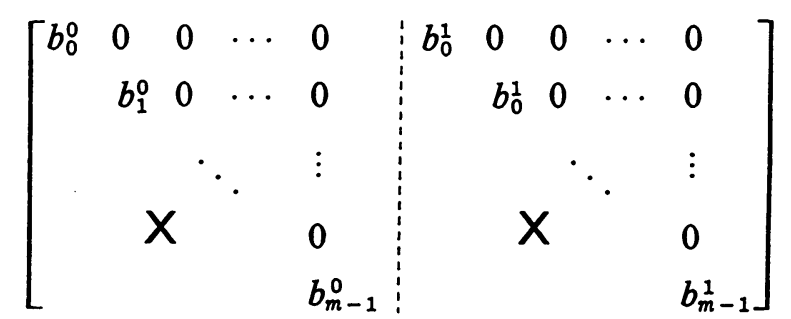

with rank $\left(b_{j}^{0}, b_{j}^{1}\right)=r_{j}$ and $\sum_{j=0}^{m-1} r_{j}=m$. The normalized conditions read

$$
b_{j}^{0} u^{(j)}(0)+\mathrm{X}+b_{j}^{1} u^{(j)}(1)+\mathrm{X}=0, \quad j=0,1, \ldots, m-1 .
$$

The crosses take the place of lower order terms at 0 and at 1 .

In illustration, if $m=4, r_{0}=r_{3}=1, r_{1}=2, r_{2}=0$, then

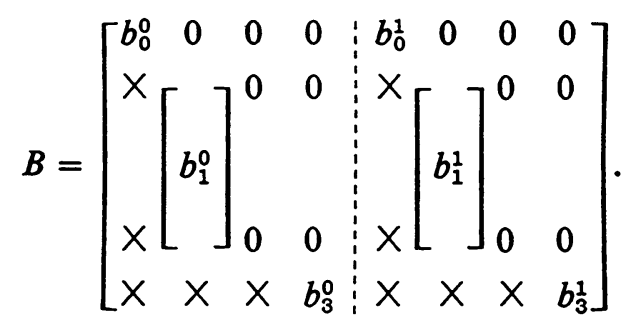

We now define the differential operator $L$, with domain $D_{L}=\left\{u \in H_{1}: b u=0\right\}$, by setting $L u=l u$. Then $L$ is a densely defined operator in $H$, and is closed [4]. 
3. The adjoint, and selfadjointness conditions. Associated with each (normalized) boundary matrix $B$ is its $m$ dimensional null space $S$, spanned by $m$ linearly independent solutions of $B x=0$. Conversely, given an $m$ dimensional subspace of $C^{2 m}$, there is an $m \times 2 m$ matrix $B$, unique to within left multiplication by an arbitrary nonsingular matrix $T$, which annihilates this subspace. A one to one correspondence between classes of equivalent boundary conditions and the subspaces associated with their matrices is established in this way. The matrix $A$ of the previous section maps $S$ onto another $m$ dimensional subspace $A S$. Let $B^{\prime}$ be a boundary matrix corresponding to $(A S)^{\perp}$, the orthogonal complement of $A S$ in $C^{2 m}$. We define a differential operator $L^{\prime}$ with $D_{L^{\prime}}=\left\{v \in H_{1}: B^{\prime} \hat{v}=0\right\}$ by putting $L^{\prime} v=l^{*} v$. Then $L^{\prime}=L^{*}$, the operator adjoint to $L$ [4].

When $D_{L}=D_{L^{*}}$, i.e. when $A S=S^{\perp}$, we say that "The conditions $b u=0$ are selfadjoint with respect to $l$ " and abbreviate this statement by "S.A." We also speak of such boundary conditions as selfadjoint, or of selfadjoint type, with the dependence on $l$ being understood.

LEMMA 1. S.A. if and only if $B A^{-1} B^{*}=0$.

Proof. Since $S$ is the null space of $B$ we have $S^{\perp}=R\left(B^{*}\right)$, the range of $B^{*}$, and $A S=S^{\perp}$ if and only if $S=R\left(A^{-1} B^{*}\right)$, if and only if $B A^{-1} B^{*}=0$.

Referring to the forms of $A$ and $B$ in 2 , we see that $A^{-1}, B^{*}$ and $B A^{-1} B^{*}$ are given, in this order, by the following three matrices.
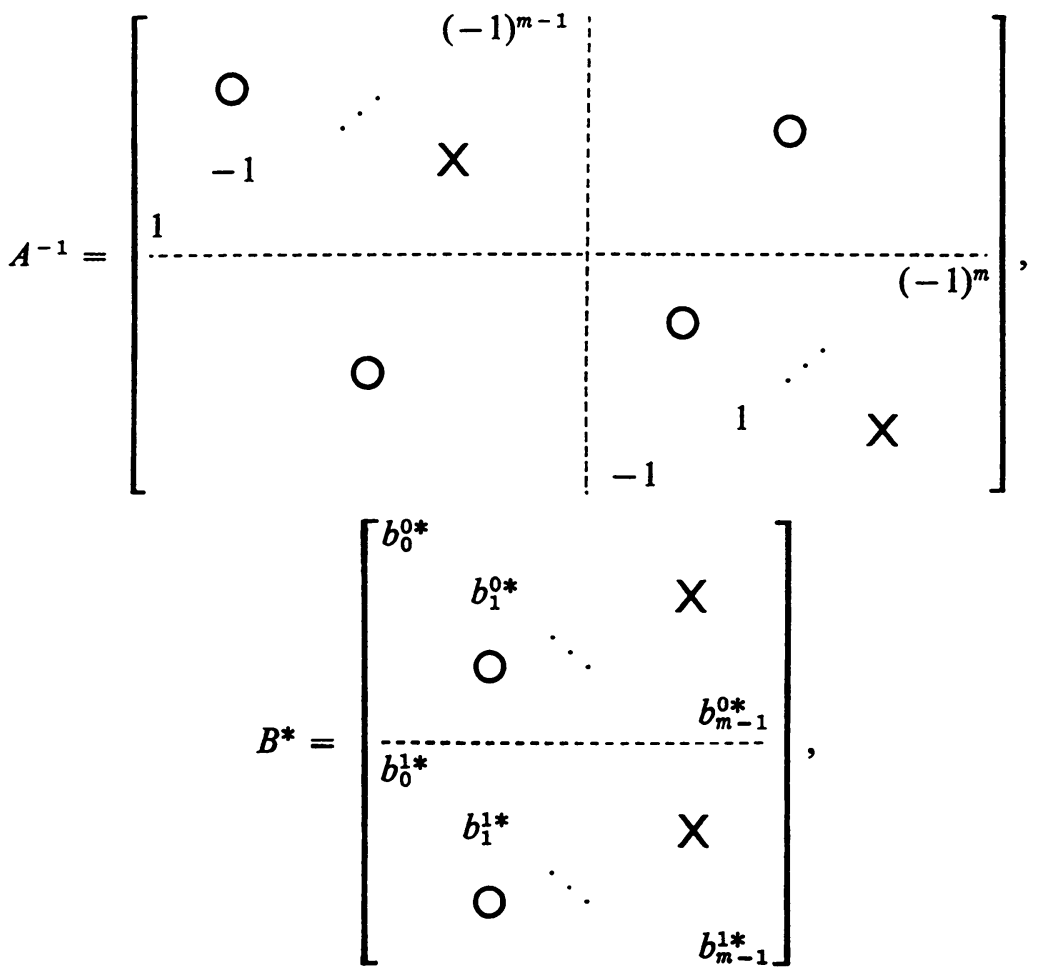
$B A^{-1} B^{*}=$

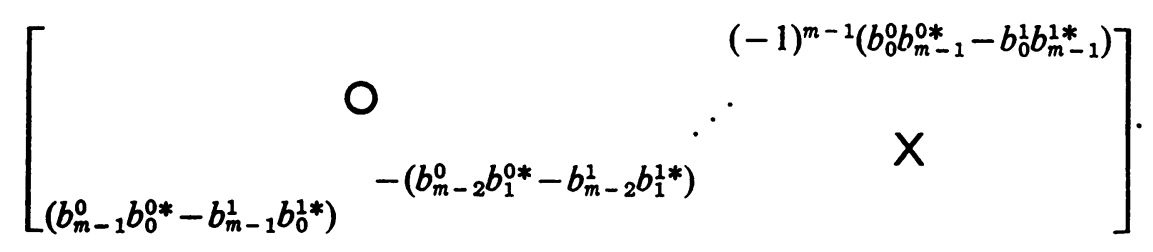

In the example,

$$
\begin{gathered}
B^{*}=\left[\begin{array}{cccc}
\bar{b}_{0}^{0} & \times & \times & \times \\
0 & \left(b_{1}^{0 *}\right) & \times \\
0 & 0 & 0 & \times \\
0 & 0 & 0 & \bar{b}_{3}^{0} \\
\bar{b}_{0}^{1} & \times & \times & \times \\
0 & \left(b_{1}^{1 *}\right) & \times \\
0 & 0 & 0 & \times \\
0 & 0 & 0 & \bar{b}_{3}^{1}
\end{array}\right], \\
B A^{-1} B^{*}=\left[\begin{array}{cccc}
0 & 0 & 0 & -b_{0}^{0} \bar{b}_{3}^{0}+b_{0}^{1} b_{3}^{1} \\
0 & 0 & 0 & \times \\
0 & 0 & 0 & \times \\
b_{3}^{0} \bar{b}_{0}^{0}-b_{3}^{1} b_{0}^{1} & \times & \times & \times
\end{array}\right] .
\end{gathered}
$$

The particular consequence of S.A. we use is

$$
b_{j}^{0} b_{m-1-j}^{0 *}=b_{j}^{1} b_{m-1-j}^{1 *}, \quad j=0,1, \ldots, m-1 .
$$

It will be understood (as the example indicates) that the $j$ th of these equations is absent if $r_{j}=0$. When $r_{j}=1$ we interpret $b_{j}^{0}, b_{j}^{1}$ as scalars.

The equations (1) are the full extent of the information we would obtain if $l u=u^{(m)}$, for in this case all terms symbolized by crosses in $A$ and $B A^{-1} B^{*}$ vanish.

LEMMA 2. If $S . A$., then for $j=0,1, \ldots, m-1$,

(i) if $r_{j}=r_{m-1-j}=1$, then $b_{j}^{0} \bar{b}_{m-1-j}^{0}=b_{j}^{1} \bar{b}_{m-1-j}^{1}$,

(ii) if $r_{j}=2$ then $r_{m-1-j}=0$,

(iii) the cases $r_{j}=r_{m-1-j}=0$, and $r_{j}=0, r_{m-1-j}=1$ cannot occur.

Proof. Equation (1) implies (i).

If $r_{j}=2$ there is a nonsingular $2 \times 2$ matrix $C$ such that

Then from (1),

$$
C b_{j}^{0}=\left(\begin{array}{l}
1 \\
0
\end{array}\right) \quad \text { and } \quad C b_{j}^{1}=\left(\begin{array}{l}
0 \\
1
\end{array}\right) \text {. }
$$

$$
\left(\begin{array}{l}
1 \\
0
\end{array}\right) b_{m-1-j}^{0 *}=\left(\begin{array}{l}
0 \\
1
\end{array}\right) b_{m-1-j}^{1 *}
$$


Hence (ii), and the implication that $r_{j}+r_{m-1-j} \leqq 2$ for each $j$. Now since $\sum_{j=0}^{m-1} r_{j}=m$, we must have $r_{j}+r_{m-1-j}=2$, and the cases in (iii) are excluded.

For use in §II, where S.A. is assumed, we suppose that $k+2 h=m$ and that $r_{f_{0}}=\cdots=r_{f_{h-1}}=2, r_{g_{0}}=\cdots=r_{g_{k-1}}=1$. Let the sequences $\left\{f_{j}\right\},\left\{g_{j}\right\}$ be strictly increasing. Then of course $g_{j}+g_{k-1-j}=m-1, j=0, \ldots, k-1$.

Also, without loss of generality the normalization can be accomplished so that

$$
\left(b_{j}^{1}, b_{j}^{0}\right)=\left(\begin{array}{ll}
1 & 0 \\
0 & 1
\end{array}\right), \quad j=f_{0}, \ldots, f_{h-1} .
$$

4. The Birkhoff expansion theorem. Birkhoff [1] defined regular boundary conditions. These entail the nonvanishing of two $m \times m$ determinants, to be defined in §II, depending only on $b_{j}^{0}, b_{j}^{1}$ but not on the expression $l$. We write " $R$ " for "The boundary conditions $b u=0$ are regular." Under assumption $R$, it can be shown [3], [10] that (i) The eigenvalues of $L$ form a denumerable sequence $\left\{\lambda_{j}\right\}$ with no finite accumulation point. The adjoint boundary conditions are also regular [8], and the eigenvalues of $L^{*}$ are $\left\{\bar{\lambda}_{j}\right\}$. (ii) Explicit asymptotic estimates, for large $|\lambda|$, can be obtained for the eigenvalues and corresponding eigenfunctions. (iii) Under the additional assumption that each eigenvalue is simple, any function $f$ in $D_{L}$ can be expanded in a uniformly convergent series

$$
f=\sum_{j=1}^{\infty}\left(f, z_{j}\right) y_{j}
$$

The $y_{j}$ and $z_{j}$ are the eigenfunctions of $L$ and $L^{*}$ corresponding to the eigenvalues $\lambda_{j}, \lambda_{j}$, and $\left(y_{j}, z_{j}\right)=1$. (iv) If the boundary conditions are 'strongly regular' (another determinantal condition if $m$ is even, one implied by the additional assumption of (iii)), the sequence $\left\{y_{j}\right\}$ is a Riesz basis in $H$, and we can normalize so that $\left(y_{j}, z_{k}\right)=\delta_{j k}[7],[2$, p. 311].

5. Statement of our theorem; remarks; acknowledgement. Writers on the Birkhoff expansion theorem have assumed $R$ when necessary for the analysis. The nature of regular boundary conditions remains largely unexplored. In particular, Kamke $[5, \mathrm{p} .197]$ noted that the question of whether selfadjoint boundary conditions are regular appeared open, except for $m=2$ where the answer is 'yes'. In this paper we prove

THEOREM. If $m$ is even, and if the boundary conditions are selfadjoint with respect to the differential expression $u^{(m)}$ then they are regular for the expression $l$.

By virtue of this theorem, and the remark after equations (1), we can assert that if $m$ is even then S.A. implies $R$.

\$II contains a simplification of the regularity determinants, and exploitation of the selfadjointness hypothesis to cast them into one standard form. This is done for both even and odd $m$. In $\S I I I$ we construct a class of positive definite matrices which will be central to the proof in $\S I V$ that the standard form is nonvanishing for even $m$. Before proceeding, we make two remarks. 
(a) A proof of the theorem for odd $m$ has not yet been found, except for $m=3$ and 5. We are hopeful, however, that the present methods can be extended to this case also $\left(^{1}\right)$.

(b) The theorem shows that if $m$ is even and $L$ is normal then $R$. This follows from the fact $\left[9\right.$, p. 33] that $D_{L}=D_{L^{*}}$ for (closed) normal operators $L$. Conditions for normality are listed in [12].

I would like to thank Professor H. O. Cordes for suggesting the problem under consideration, and for showing me the close relation between Lemma III and the proof of the theorem. I am also grateful to Professors Donald Sarason and David Spring for many useful discussions.

\section{Simplification of the regularity determinants.}

1. Replacement of $\omega_{j}$ by $\varepsilon_{j}$. Defining regularity [3], we must distinguish case (E), $m$ even, and case (O), $m$ odd. In (E), $m=2 q$ and the boundary conditions $b u=0$ are regular if $\theta_{0} \neq 0$ and $\theta_{2} \neq 0$ in the identity

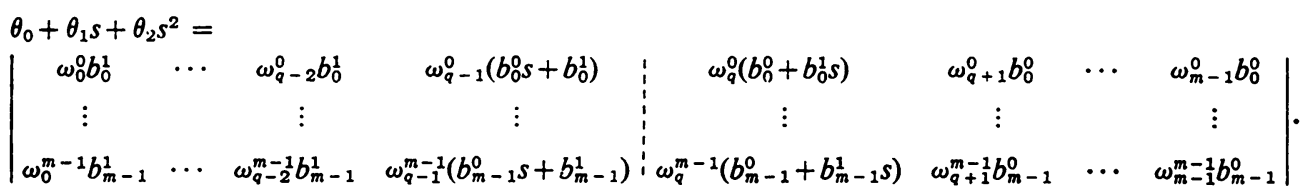

In (O), $m=2 q+1$ and we have regularity if $\theta_{0} \neq 0, \theta_{1} \neq 0$ in the identity $\theta_{0}+\theta_{1} s=$

$\left|\begin{array}{ccccccc}\omega_{0}^{0} b_{0}^{1} & \cdots & \omega_{q-1}^{0} b_{0}^{1} & \omega_{q}^{0}\left(b_{0}^{0}+b_{0}^{1} s\right) & \omega_{q+1}^{0} b_{0}^{0} & \cdots & \omega_{m-1}^{0} b_{0}^{0} \\ \vdots & & \vdots & \vdots & \vdots & & \vdots \\ \omega_{0}^{m-1} b_{m-1}^{1} & \cdots & \omega_{q-1}^{m-1} b_{m-1}^{1} & \omega_{q}^{m-1}\left(b_{m-1}^{0}+b_{m-1}^{1} s\right) & \omega_{a+1}^{m-1} b_{m-1}^{0} & \cdots & \omega_{m-1}^{m-1} b_{m-1}^{0}\end{array}\right|$.

The sequence of distinct unit roots $\omega_{0}, \omega_{1}, \ldots, \omega_{m-1}$ is a permutation of the naturally ordered sequence $\varepsilon_{0}, \varepsilon_{1}, \ldots, \varepsilon_{m-1}$, with $\varepsilon_{j}=e^{2 \pi i j / m}: \omega_{0}=\varepsilon_{0}=1, \omega_{1}=\varepsilon_{m-1}$, $\omega_{2}=\varepsilon_{1}, \omega_{3}=\varepsilon_{m-2}, \ldots, \omega_{2 j}=\varepsilon_{j}, \omega_{2 j+1}=\varepsilon_{m-1-j}, \ldots$

We are going to show that the numbers $\omega_{j}$ can be replaced by $\varepsilon_{j}$ in the determinants $\theta_{0}, \theta_{1}$ and $\theta_{2}$. In (E) this will enable us to prove that $\theta_{0}= \pm \theta_{2}$. A simplification will also be possible in $(\mathrm{O})$. We define the standard form

$$
\theta=\left|\begin{array}{cccccc}
\varepsilon_{0}^{0} b_{0}^{1} & \cdots & \varepsilon_{q-1}^{0} b_{0}^{1} & \varepsilon_{q}^{0} b_{0}^{0} & \cdots & \varepsilon_{m-1}^{0} b_{0}^{0} \\
\vdots & & \vdots & \vdots & & \vdots \\
\varepsilon_{0}^{m-1} b_{m-1}^{1} & \cdots & \varepsilon_{q-1}^{m-1} b_{m-1}^{1} & \varepsilon_{q}^{m-1} b_{m-1}^{0} & \cdots & \varepsilon_{m-1}^{m-1} b_{m-1}^{0}
\end{array}\right| .
$$

This definition is operative for both (E) and (O). The first $q$ columns of $\theta$ involve $b^{1}$ (by which is meant $b_{j}^{1}, j=0,1, \ldots, m-1$ ). In (E), the last $q$ involve $b^{0}$ and in (O) this number is $q+1$. Write $\theta=\theta\left(b^{0}, b^{1}\right)$ when it is necessary to emphasize that the first argument appears on the right, the second on the left.

LEMMA. $R$ if and only if (E) $\theta \neq 0$;

(O) $\theta\left(b^{0}, b^{1}\right) \neq 0$ and $\theta\left(b^{1}, b^{0}\right) \neq 0$.

( $\left.{ }^{1}\right)$ Added in proof. We have recently obtained a proof for odd $m$. 
Proof. We begin with (E). That fact that $b^{1}$ appears only on the left and $b^{0}$ only on the right of $\theta_{0}$ will not be altered if we execute certain interchanges among the first $q$ columns, and also among the last $q$. The particulars depend on whether $q$ is even or odd.

(a) $q$ is even. The interchange is indicated in the following scheme. The first line consists of the column subscripts of the $\omega_{j}$ prior to the interchange, while the second contains the new subscripts (Figure 1).

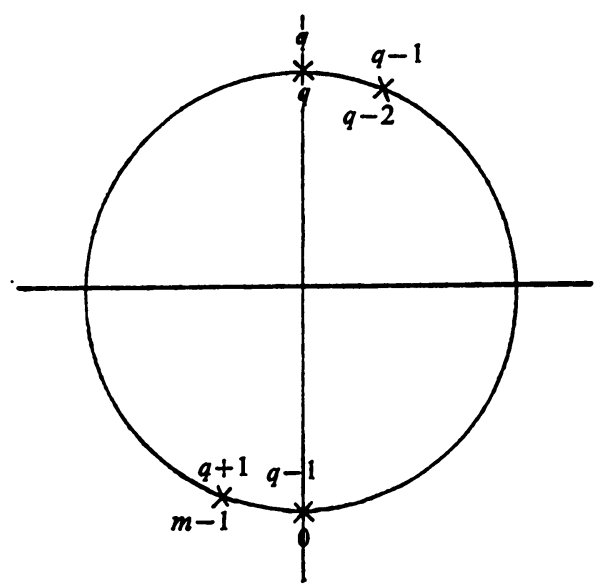

$$
\begin{array}{cccccccc}
\cdots & q-3 & q-2 & q-1 & q & q+1 & q+2 & \cdots \\
1 & q-1 & 0 & q & m-1 & q+1 & \\
\multicolumn{6}{c}{\text { FIGURE } 1} & &
\end{array}
$$

(b) $q$ odd (Figure 2).

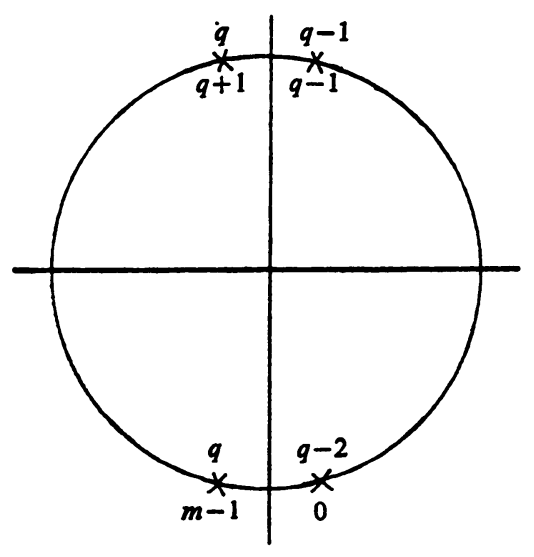

$$
\begin{aligned}
& \begin{array}{llllllll}
\cdots & q-3 & q-2 & q-1 & q & q+1 & q+2 & \cdots
\end{array} \\
& q-2 \quad 0 \quad q-1 \quad m-1 \quad q \quad m-2
\end{aligned}
$$


The new ordering, as shown by the integers outside the circumference in Figures 1 and 2 , is cyclic in the counterclockwise sense. Putting

$$
\begin{aligned}
\phi & =\omega_{q-1}, \quad q \text { even, } \\
& =\omega_{q-2}, \quad q \text { odd },
\end{aligned}
$$

we see that $\theta$ is obtained from $\theta_{0}$ by interchanging columns and replacing each $\varepsilon_{j}$ by $\phi \varepsilon_{j}$. Hence

$$
\theta_{0}= \pm \theta
$$

In $\theta_{2}$, first interchange the center columns $q-1$ and $q$, so that $b^{1}$ appears only on the left, and $b^{0}$ only on the right.

(a) $q$ even

(b) $q$ odd

$$
\begin{array}{cccccccc}
\cdots & q-3 & q-2 & q-1 & q & q+1 & q+2 & \cdots \\
0 & q-2 & m-1 & q-1 & m-2 & q &
\end{array}
$$

and again

$$
\begin{aligned}
& \begin{array}{lllllllll}
\cdots & q-3 & q-2 & q-1 & q & q+1 & q+2 & \cdots
\end{array} \\
& q-1 \quad 1 \quad q \quad 0 \quad q+1 \quad m-1
\end{aligned}
$$

Taking up (O), we look first at $\theta_{0}$.

(a) $q$ even

(b) $q$ odd

$$
\begin{aligned}
& \begin{array}{lllllllll}
\cdots & q-3 & q-2 & q-1 & q & q+1 & q+2 & \cdots
\end{array} \\
& \begin{array}{llllll}
1 & q-1 & 0 & q & m-1 & q+1
\end{array}
\end{aligned}
$$

$$
\begin{array}{rcccccc}
\cdots & q-3 & q-2 & q-1 & q & q+1 & q+2 \\
& q-2 & 0 & q-1 & m-1 & q & m-2
\end{array}
$$

$$
\theta_{0}= \pm \theta\left(b^{0}, b^{1}\right) \text {. }
$$

Passing finally to $\theta_{1}$, we transfer the last $q$ columns, those containing $b^{0}$, to the left.

(a) $q$ even

(b) $q$ odd

$$
\begin{array}{cccccccc}
\cdots & q-3 & q-2 & q-1 & q & q+1 & q+2 & \cdots \\
& q+1 & m-2 & q & m-1 & q-1 & 0 &
\end{array}
$$

$$
\begin{array}{cccccccc}
\cdots & q-3 & q-2 & q-1 & q & q+1 & q+2 & \cdots \\
m-2 & q+1 & m-1 & q & 0 & q-1 &
\end{array}
$$

$$
\theta_{1}= \pm \theta\left(b^{1}, b^{0}\right) \text {. }
$$

Equations (1)-(4) establish the lemma. 
2. Treatment of $r_{j} \neq 1$. We must come to grips with the fact that some of the matrices $\left(b_{j}^{1}, b_{j}^{0}\right)$ may be empty. Consult $\S I .3$ for the definition of $f_{j}$ and $g_{j}$. Let $L$ be the ordered set $\left\{g_{0}, \ldots, g_{l-1}\right\}$, where $k=2 l$ in (E) and $k=2 l+1$ in (O). Also, let

$$
\left(b_{g_{j}}^{1}, b_{g_{j}}^{0}\right)=\left(\beta_{g_{j}}, \alpha_{g_{j}}\right),\left|\alpha_{g_{j}}\right|+\left|\beta_{g_{j}}\right|>0, \quad j=0,1, \ldots, k-1 .
$$

Shift all the rows of $\theta$ corresponding to the $f_{j}$ to the top, so that

$$
\theta= \pm\left|\begin{array}{cccccc}
\varepsilon_{f_{0}}^{0} & \cdots & \varepsilon_{f_{0}}^{q-1} & 0 & \cdots & 0 \\
0 & \cdots & 0 & e_{f_{0}}^{q} & \cdots & \varepsilon_{f_{0}}^{m-1} \\
& \cdots & & & \cdots & \\
\varepsilon_{f_{h-1}}^{0} & \cdots & \varepsilon_{f_{h}-1}^{q-1} & 0 & \cdots & 0 \\
0 & \cdots & 0 & \varepsilon_{f_{h-1}}^{q} & \cdots & \varepsilon_{f_{h-1}^{m}}^{m-1} \\
\beta_{g_{0}} \varepsilon_{g_{0}}^{0} & \cdots & \beta_{g_{0}} \varepsilon_{g_{0}}^{q-1} & \alpha_{g_{0}} \varepsilon_{g_{0}}^{q} & \cdots & \alpha_{g_{0}} \varepsilon_{g_{0}}^{m-1} \\
\vdots & & \vdots & \vdots & & \vdots \\
\beta_{g_{k-1}} \varepsilon_{g_{k-1}}^{0} & \cdots & \beta_{g_{k-1}} \varepsilon_{g_{k-1}-1}^{q-1} & \alpha_{g_{k-1}} \varepsilon_{g_{k-1}}^{q} & \cdots & \alpha_{g_{k-1}} \varepsilon_{g_{k-1}-1}^{m-1}
\end{array}\right| .
$$

LEMMA.

$$
\theta=c\left[\prod_{0 \leqq j<j^{\prime} \leqq h-1}\left(\varepsilon_{f_{j^{\prime}}}-\varepsilon_{f_{j}}\right)^{2} \prod_{j=0 ; j^{\prime}=0}^{k-1 ; h-1}\left(\varepsilon_{g_{j^{\prime}}}-\varepsilon_{f_{j}}\right)\right] \theta_{L},
$$

where $|c|=1$ and

$$
\theta_{L}=\left|\begin{array}{cccccc}
\beta_{g_{0}} \varepsilon_{g_{0}}^{0} & \cdots & \beta_{g_{0}} \varepsilon_{g_{0}}^{l-1} & \alpha_{g_{0}} \varepsilon_{g_{0}}^{q} & \cdots & \alpha_{g_{0}} \varepsilon_{g_{0}}^{m+l-q-1} \\
\vdots & & \vdots & \vdots & & \vdots \\
\beta_{g_{k-1}} \varepsilon_{g_{k-1}}^{0} & \cdots & \beta_{g_{k-1}} \varepsilon_{g_{k-1}-1}^{l-1} & \alpha_{g_{k-1}} \varepsilon_{g_{k-1}}^{q} & \cdots & \alpha_{g_{k-1}} \varepsilon_{g_{k-1}}^{m+l-q-1}
\end{array}\right|
$$

Proof. In the formula for $\theta$, empty products, occurring if $h=0$ or 1 , or if $k=0$, will be interpreted as equal to one. We will refer to the columns of $\theta$ by the exponent of $\varepsilon_{g_{1}}$ they carry. Perform the following column operations on $\theta$. Multiply column $q-1-j$ by $\varepsilon_{f_{0}}$ and subtract from column $q-j$, for $j=1, \ldots, q-1$. Multiply column $m-1-j$ by $\varepsilon_{f_{0}}$ and subtract from column $m-j$, for $j=1, \ldots, m-1-q$.

Expand the resulting determinant from its first two rows, and then extract the factor

$$
\prod_{j=1}^{h-1}\left(\varepsilon_{f_{j}}-\varepsilon_{f_{0}}\right)^{2} \prod_{j=0}^{k-1}\left(\varepsilon_{g_{j}}-\varepsilon_{f_{0}}\right)
$$

The determinant remaining is of order $m-2$. We got it from $\theta$ by crossing out rows 0 and 1 , and columns $q-1$ and $m-1$. This process is to be carried out $h$ times, leading to the lemma. Thus $\theta_{L}$ is obtained from $\theta$ by crossing out the first $2 h$ rows, and columns $q-j, m-j, j=1, \ldots, h$. We write $\theta_{L}=\theta_{L}(\alpha, \beta)$ to stress that the columns on the right contain the $\alpha_{g}$. 
COROLLARY. The boundary conditions $b u=0$ are regular if and only if

(E) $\theta_{L} \neq 0$;

(O) $\theta_{L}(\alpha, \beta) \neq 0$ and $\theta_{L}(\beta, \alpha) \neq 0$.

The corollary follows from the above and Lemma 1.

3. Use of selfadjoint type. If S.A. then

$$
\alpha_{g_{j}} \bar{\alpha}_{g_{k-1-j}}=\beta_{g} \bar{\beta}_{g_{k-1-j}}, \quad j=0,1, \ldots, k-1,
$$

an immediate consequence of Lemma I.2.

Once more, we are forced to distinguish cases (E) and (O).

In (E), $k=2 l$ and

$$
\alpha_{g}, \bar{\alpha}_{g_{2 l-1-j}}=\beta_{g,} \bar{\beta}_{2 l-1-\jmath}, \quad j=0,1, \ldots, l-1 .
$$

Multiply the last row of $\theta_{L}$ by $\bar{\alpha}_{g_{0}}$ and then remove the factor $\beta_{g_{2 l-1}}$ from that row. Next multiply the same row by $\bar{\beta}_{g_{0}}$ and factor $\alpha_{g_{2 l-1}}$. Writing $\theta_{L}^{\prime}$ for the determinant whose last row is

$$
\bar{\alpha}_{g_{0}} \varepsilon_{m-1-g_{0}}^{0} \cdots \bar{\alpha}_{g_{0}} \varepsilon_{m-1-g_{0}}^{l-1} \quad \bar{\beta}_{g_{0}} \varepsilon_{m-1-g_{0}}^{q} \cdots \bar{\beta}_{g_{0}} \varepsilon_{m-1-g_{0}}^{q+l-1}
$$

and which agrees with $\theta_{L}$ in all other rows, we have

$$
\bar{\alpha}_{g_{0}} \theta_{L}=\beta_{g_{2 l-1}} \theta_{L}^{\prime} \quad \text { and } \quad \bar{\beta}_{g_{0}} \theta_{L}=\alpha_{g_{2 l-1}} \theta_{L}^{\prime} .
$$

Thus

$$
\left(\left|\alpha_{g_{0}}\right|+\left|\beta_{g_{0}}\right|\right)\left|\theta_{L}\right|=\left(\left|\alpha_{g_{2 l-1}}\right|+\left|\beta_{g_{2 l-1}}\right|\right)\left|\theta_{L}^{\prime}\right| \text {. }
$$

Doing this to each of the bottom $l$ rows of $\theta_{L}$, and abbreviating

$$
\delta_{L}=\prod_{j=0}^{l-1}\left(\left|\alpha_{g_{2 l-1-j}}\right|+\left|\beta_{g_{2 l-1-j}}\right|\right) /\left(\left|\alpha_{g_{j}}\right|+\left|\beta_{g_{j}}\right|\right),
$$

so that $\delta_{L}>0$, we get

where

$$
\left|\theta_{L}\right|=\delta_{L}|D|
$$

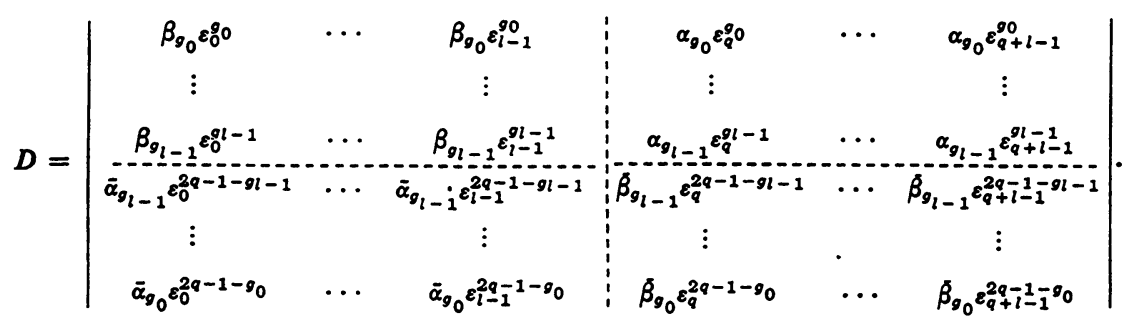

Combining this with Corollary 2, we have

Proposition E. Boundary conditions of selfadjoint type are regular in case (E) if for arbitrary complex numbers $\alpha_{j}, \beta_{j}$, subject only to the restriction that $\left|\alpha_{j}\right|+\left|\beta_{j}\right|>0, j \in L$, we have $D \neq 0$. 
In (O), $m=2 q+1$, we have (5), and in addition

$$
\alpha_{q} \bar{\alpha}_{q}=\beta_{q} \bar{\beta}_{q} \neq 0 \text {. }
$$

It will be convenient to assume $\left|\alpha_{q}\right|=\left|\beta_{q}\right|=1$, and put $\alpha_{q}=e^{i \psi}, \beta_{q}=e^{i \chi}, \psi$ and $\chi$ real.

Employing the method of $(\mathrm{E})$, and the $\delta_{L}$ defined there,

where

$$
\left|\theta_{L}(\alpha, \beta)\right|=\delta_{L}\left|D^{\prime}(\alpha, \beta)\right|,
$$

$$
D^{\prime}(\alpha, \beta)=\left|\begin{array}{clllll}
\beta_{g_{0}} \varepsilon_{0}^{q} 0 & \cdots & & & & \\
\vdots & & & & & \\
\beta_{g_{l-1}} \varepsilon_{0}^{g_{l-1}} & \cdots & & & & \\
e^{i \psi} \varepsilon_{0}^{q} & \cdots & e^{i \psi} \varepsilon_{l-1}^{q} & e^{i \chi} \varepsilon_{q}^{q} & \cdots & e^{i x} \varepsilon_{q+l}^{q} \\
\bar{\alpha}_{g_{l-1}-1} \varepsilon_{0}^{2 q-g_{l-1}} & \cdots & & & & \\
\vdots & & & & & \\
\bar{\alpha}_{g_{0}} \varepsilon_{0}^{2 q-g_{0}} & \cdots & & & &
\end{array}\right| .
$$

Multiply each of the first $l$ columns of $D^{\prime}(\alpha, \beta)$ by $e^{i(x-\psi) / 2}$.

Multiply each of the last $l+1$ columns of $D^{\prime}(\alpha, \beta)$ by $e^{i(\psi-x) / 2}$.

The factor $e^{i(\psi+x) / 2}$ can be removed from the middle row of the resulting determinant. Changing variables

$$
\alpha_{j}^{\prime}=e^{i(\psi-x) / 2} \alpha_{j}, \quad \beta_{j}^{\prime}=e^{i(x-\psi) / 2} \beta_{j}, \quad j \in L,
$$

we get $D^{\prime}(\alpha, \beta)=e^{i x} D\left(\alpha^{\prime}, \beta^{\prime}\right)$, where $D$ agrees with $D^{\prime}$ except that its middle row is

\section{Recapitulating,}

$$
\varepsilon_{0}^{q} \cdots \varepsilon_{q+l}^{q} \text {. }
$$

PROPOSITION O. Boundary conditions of selfadjoint type are regular in case $(0)$ if for arbitrary complex numbers $\alpha_{j}, \beta_{j}$, subject only to the restriction that $\left|\alpha_{j}\right|+\left|\beta_{j}\right|>0$, $j \in L$, we have $D(\alpha, \beta) \neq 0$.

Proof. The inequality $D(\alpha, \beta) \neq 0$ implies that $D\left(\alpha^{\prime}, \beta^{\prime}\right) \neq 0$, and since $\alpha_{j}, \beta_{j}$ are arbitrary, that $D\left(\beta^{\prime}, \alpha^{\prime}\right) \neq 0$. Corollary 2 now applies.

In closing this section, we shift back to case $(E)$, where we will remain until the conclusion. The theorem of this paper will be established when Lemma IV is proved. The forthcoming Lemma III will be auxiliary to this proof.

III. A class of positive definite matrices. In this section $C_{k}(A)$ will denote the $k$ th compound of the matrix $A$, with the property that $C_{k}(A B)=C_{k}(A) C_{k}(B)$. [6, pp. 16-17].

1. A brief review of positive definiteness. The hermitian matrix $A=\left(a_{j k}\right)_{j, k=1}^{n}$ is p.d. if $(A x, x)=\sum_{j, k=1}^{n} a_{j k} x_{j} \bar{x}_{k}>0$ for all nonzero vectors $x=\left(x_{1}, \ldots, x_{n}\right)$. It is a consequence that $A$ is p.d. if and only if all its principal minors (the ones chosen from the same rows as columns) are positive, or if and only if $A=B^{2}$ for some nonsingular hermitian $B$. If $A$ and $B$ are hermitian and $a_{j k}=d_{j} d_{k}^{\prime} b_{j k}$, where $d_{j}, d_{k}^{\prime}$ are positive, $j, k=1, \ldots, n$, then $A$ is p.d. if and only if $B$ is. 
If $A$ is p.d. then so are $A \otimes A$ and $C_{k}(A), k=1, \ldots, n$. In fact $B^{2} \otimes B^{2}=(B \otimes B)^{2}$ and $C_{k}\left(B^{2}\right)=\left(C_{k}(B)\right)^{2}$. The matrix of squares $\left(a_{j k}^{2}\right)_{j, k=1}^{n}$, being a principal submatrix of $A \otimes A$, is p.d. also.

2. A class of positive definite matrices. We require a notation for matrices indexed by sets somewhat more general than the natural numbers. Let $Q$ be the ordered set $\{0,1, \ldots, q-1\}$. For $r=1, \ldots, q, Q_{r}$ is the set of all strictly increasing sequences in $Q$ of length $r ; \rho$ and $\sigma$ will always be elements of $Q_{r}$. Order $Q_{r}$ by setting $\rho>\sigma$ if the first integer in $\rho$ which differs from the corresponding integer in $\sigma$ is greater than that integer. Let $\left(a_{\rho \sigma}\right)_{\rho, \sigma \in Q_{r}}$ be the $\left(\begin{array}{l}q \\ r\end{array}\right) \times\left(\begin{array}{l}q \\ r\end{array}\right)$ matrix whose elements $a_{\rho \sigma}$ are indexed by $Q_{r}$ with the given ordering. Thus $\left(a_{j k}\right)_{j, k=1}^{q-1}$, indexed by $Q_{1}$, is the usual notation for a $q \times q$ matrix. Its $r \times r$ submatrix with row and column indices in $\rho$ and $\sigma$ respectively, is written $\left(a_{j k}\right)_{j \in \rho, k \in \sigma}$.

LeMma III. The matrices $P_{r}$ are positive definite for $r=1,2, \ldots, q$, where

$$
P_{r}=\left(\prod_{j \in \rho ; k \in \sigma} \operatorname{cosec}\left(x_{j}+x_{k}\right)\right)_{0, \sigma \in Q_{r}}
$$

and $0<x_{0}<x_{1}<\cdots<x_{q-1}<\pi / 2$.

Proof. First observe that each $P_{r}$ is a symmetric matrix with positive elements. Now fix $r, 2 \leqq r \leqq q$. We define the positive quantities

$$
\begin{aligned}
t_{j} & =\tan x_{j}, \quad j \in Q, \quad T_{o}=\prod_{j^{\prime}>\sum_{j}, j_{j} \in \rho}\left(t_{j^{\prime}}-t_{j}\right), \\
C_{o} & =\prod_{j \in \rho} \cos x_{j}, \quad y_{\rho \sigma}=\prod_{j \in \rho ; k \in \sigma} \operatorname{cosec}\left(x_{j}+x_{k}\right) .
\end{aligned}
$$

Let $p_{\rho \sigma}$ be the minor of $P_{1}$ with row and column indices in $\rho$ and $\sigma$. Then

$$
\begin{aligned}
p_{\rho \sigma} & =\operatorname{det}\left(\operatorname{cosec}\left(x_{j}+x_{k}\right)\right)_{j \in \rho, k \in \sigma} \\
& =\operatorname{det}\left(\sec x_{j} \sec x_{k}\left(t_{j}+t_{k}\right)^{-1}\right)_{j \in \rho, k \in \sigma} \\
& =C_{\rho}^{-1} C_{\sigma}^{-1} \operatorname{det}\left(\left(t_{j}+t_{k}\right)^{-1}\right)_{j \in \rho, k \in \sigma} \\
& =C_{\rho}^{-1} C_{\sigma}^{-1} T_{\rho} T_{\sigma} \prod_{j \in \rho ; k \in \sigma}\left(t_{j}+t_{k}\right)^{-1} \\
& =C_{\rho}^{r-1} C_{\sigma}^{r-1} T_{\rho} T_{\sigma} y_{\rho \sigma} .
\end{aligned}
$$

The second equation is a consequence of the identity $\operatorname{cosec}(x+y)=\sec x \sec y$ $\cdot(\tan x+\tan y)^{-1}$, and the fourth holds by a lemma of Cauchy [10, Volume 2, pp. 98, 299]. It follows that $p_{\rho \sigma}$ is positive and that

$$
P_{r}=D_{r} C_{r}\left(P_{1}\right) D_{r} .
$$

Here $D_{r}$ is a diagonal matrix with positive diagonal elements $C_{\rho}^{1-r} T_{\rho}^{-1}, \rho \in Q_{r}$.

In conclusion, $P_{1}$ is p.d. (it is even totally definite). Thus $C_{r}\left(P_{1}\right)$ is p.d., and from (1), $P_{r}$ is p.d., $r=2, \ldots, q$. 
We remark that this proof generalizes easily to show that if

(i) $g$ and $h$ are positive functions defined for the sequence $x_{0}<x_{1}<\cdots<x_{q-1}$ and $h$ is strictly increasing.

(ii) $f$ satisfies the functional equation $f(x+y)=g(x) g(y)[h(x)+h(y)]^{-1}$ then

$$
\left(\prod_{j \in \rho ; k \in \sigma} f\left(x_{j}+x_{k}\right)\right)_{\rho, \sigma \in Q}
$$

is p.d. for $r=1, \ldots, q$.

IV. Proof that $D \neq 0$.

1. Some facts about the determinant $\delta$. Let $\delta$ denote the $2 l \times 2 l$ determinant obtained from $D$ by setting $\alpha_{j}=\beta_{j}=1, j \in L$.

The first $l$ columns of $\delta$ will be called the left columns and the last $l$ the right columns. Likewise, the first and last $l$ rows will be top and bottom. Let $g_{0} \leqq j_{0}$ $<j_{1}<\cdots<j_{l-1} \leqq 2 q-1-g_{0}$ and let

$$
M=\left|\begin{array}{lll}
\varepsilon_{q}^{j_{0}} & \cdots & \varepsilon_{q+l-1}^{j_{0}} \\
\vdots & & \vdots \\
\varepsilon_{q}^{j_{l-1}} & \cdots & \varepsilon_{q+l-1}^{j_{l-1}}
\end{array}\right|
$$

be a minor of $\delta$ from the right. Then

$$
M=(-1)^{j_{0}+\cdots+j_{l-1}}\left|\begin{array}{lll}
\varepsilon_{0}^{j_{0}} & \cdots & \varepsilon_{l-1}^{j_{0}} \\
\vdots & & \vdots \\
\varepsilon_{0}^{j_{l-1}} & \cdots & \varepsilon_{l-1}^{j_{l-1}}
\end{array}\right| .
$$

The latter determinant, to be called $m$, is the left minor of $\delta$ chosen from the same rows as $M$. By the relation $\varepsilon_{t}^{s}=\varepsilon_{s}^{t}, m$ is the Vandermonde

$$
m=\prod_{0 \leqq k<k^{\prime} \leqq l-1}\left(\varepsilon_{j_{k^{\prime}}}-\varepsilon_{j_{k}}\right) \text {. }
$$

In evaluating this product, we will make use of the identity

$$
\varepsilon_{t}-\varepsilon_{s}=2 i \sin \frac{\pi}{2 q}(t-s) e^{(i \pi / 2 q)(t+s)}
$$

Let $d_{1}, d_{2}, d_{3}, d_{4}$ be the 'corner' minors of $\delta$ :

$$
\delta=\left|\begin{array}{c:c}
d_{1} & d_{2} \\
\hdashline d_{3} & d_{4}
\end{array}\right|
$$

so that

$$
d_{1}=\left|\begin{array}{lll}
\varepsilon_{0}^{g} & \cdots & \varepsilon_{l-1}^{g_{0}} \\
\vdots & & \vdots \\
\varepsilon_{0}^{g_{l-1}} & \cdots & \varepsilon_{l-1}^{g_{l-1}}
\end{array}\right|, \text { etc. }
$$


Then writing $G=\sum_{j \in L} j$ and $S=\prod_{j^{\prime}>j: j ; j^{\prime} \in L} \sin (\pi / 2 q)\left(j^{\prime}-j\right)$, we get from (1), (2) and (3) that

$$
d_{1}=\prod_{j^{\prime}>j ; j, j^{\prime} \in L}\left(\varepsilon_{j^{\prime}}-\varepsilon_{j}\right)=(2 i)^{l(l-1) / 2} S e^{(i \pi / 2 q)(l-1) G}
$$

and

$$
d_{4}=(-1)^{l+G}(2 i)^{l(l-1) / 2} S e^{(i \pi / 2 q)(1-l)(l+G)} .
$$

Thus

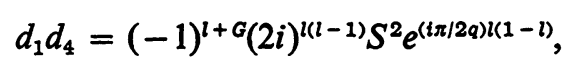

and employing (1),

$$
d_{2} d_{3}=(-1)^{l} d_{1} d_{4} .
$$

2. Proof that $D \neq 0$. For $r=1,2, \ldots, l$ we define $L_{r}$ to be the set of all strictly increasing sequences in $L$ of length $r$, and give $L_{r}$ the ordering of $Q_{r}$ (III.2). Then $L_{r} \subset Q_{r}$ and $\left(y_{\rho \sigma}\right)_{\rho, \sigma \in L_{r}}$, a principal submatrix of $P_{r}$, is p.d. This will be basic in the proof to follow.

LEMMA IV. For arbitrary complex numbers $\alpha_{j}, \beta_{j}$, subject only to the restriction that $\left|\alpha_{j}\right|+\left|\beta_{j}\right|>0, j \in L$, the $2 l \times 2 l$ determinant $D$ of II. 3 does not vanish.

Proof. The mapping ^ $: L \rightarrow\{0,1, \ldots, l-1\}$ will send $j \in L$ onto $j$ determined uniquely by $j=g_{j}$. For $j \in L$ we define $n_{j}=j+\hat{j}, N=\sum_{j \in L} n_{j}$ and $\hat{\beta}_{j}=(-1)^{n_{j}} \beta_{j}$.

The integer $r$ will now be fixed, $1 \leqq r \leqq l-1$. The symbols $\rho$ and $\sigma$ will always denote elements of $L_{r}$. We make the following definitions:

$$
\begin{aligned}
N_{o} & =\sum_{j \in \rho} n_{j,}, \\
x_{j} & =\left(j+\frac{1}{2}\right) \pi / 2 q, \quad j \in \rho, \\
S_{\rho} & =\prod_{j^{\prime}>j} \sin \left(x_{j^{\prime}}-x_{j}\right), \\
S_{\rho}^{\prime} & =S_{\rho} \prod_{j \in \rho ; k \in L} \sin \left(x_{j}+x_{k}\right), \\
c & =(-1)^{l+N} 2^{l(l-1)} e^{(\pi \pi / 2 q)(1-l)} .
\end{aligned}
$$

A second mapping $\sim: L_{r} \rightarrow L_{l-r}$ sends $\rho$ onto $\tilde{\rho} \in L_{l-r}$ consisting of those $l-r$ integers in $L$ disjoint from $\rho$. The minor $m_{\rho \sigma}$ is defined to be

$$
\left|\begin{array}{lll}
\varepsilon_{0}^{j_{0}} & \cdots & \varepsilon_{l-1}^{j_{0}} \\
\vdots & & \vdots \\
\varepsilon_{0}^{j_{r}-1} & \cdots & \varepsilon_{l-1}^{j_{r}-1} \\
\varepsilon_{0}^{2 q-1-k_{l-r-1}} & \cdots & \varepsilon_{l-1}^{2 q-1-k_{l-r-1}} \\
\vdots & & \vdots \\
\varepsilon_{0}^{2 q-1-k_{0}} & \cdots & \varepsilon_{l-1}^{2 q-1-k_{0}}
\end{array}\right|
$$


where $\rho=\left\{j_{0}, \ldots, j_{r-1}\right\}$ and $\tilde{\sigma}=\left\{k_{0}, \ldots, k_{l-r-1}\right\}$. In words, $m_{\rho \sigma}$ is the left minor of $\delta$ chosen from the rows indicated by $\rho$ in the top half and the ones indicated by $\tilde{\sigma}$, in reverse order, in the bottom half. Let $M_{\rho \sigma}$ be the cofactor of $m_{\rho \sigma}$ in $\delta$. We then have the formula

$$
m_{\rho \sigma} M_{\rho \sigma}=m_{\sigma \rho} M_{\sigma \rho}=(-1)^{N_{\rho}+N_{\sigma}} S_{\rho}^{\prime} S_{\tilde{\rho}} S_{\sigma}^{\prime} S_{\tilde{\sigma}} y_{\rho \sigma}^{2} .
$$

To prove (4) define

$$
y_{\rho \tilde{\sigma}}=\prod_{j \in \rho ; k \in \tilde{\sigma}} \operatorname{cosec}\left(x_{j}+x_{k}\right)=y_{\tilde{\sigma} \rho} .
$$

Since $y_{\rho \tilde{\sigma}} y_{\rho \sigma}=\prod_{j \in \rho: k \in L} \operatorname{cosec}\left(x_{j}+x_{k}\right)$, we get

$$
S_{\rho} y_{\rho \sigma}^{-1}=S_{\rho}^{\prime} y_{\rho \sigma} \text {. }
$$

Now using (2) and (3),

$$
m_{\rho \sigma}=(2 i)^{l(l-1) / 2} \Pi_{1} \Pi_{2} \Pi_{3},
$$

where

$$
\begin{aligned}
\Pi_{1} & =\prod_{j^{\prime}>j ; j ;, j^{\prime} \in \rho} \sin \left(x_{j^{\prime}}-x_{j}\right) e^{(i \pi / 2 q)\left(j+j^{\prime}\right)}=S_{\rho} \exp \left[\frac{i \pi}{2 q}(r-1) \sum_{j \in \mathcal{O}} j\right] \\
\Pi_{2} & =\prod_{k^{\prime}>k ; k, k k^{\prime} \in \tilde{\sigma}} \sin \left(x_{k^{\prime}}-x_{k}\right) e^{(i \pi / 2 q)\left(4 q-2-k-k^{\prime}\right)} \\
& =S_{\tilde{\sigma}} \exp \left[\frac{i \pi}{2 q}(r+1-l) \sum_{k \in \tilde{\sigma}}(k+1)\right], \\
\Pi_{3} & =\prod_{j \in \rho ; k \in \tilde{\sigma}} \sin \left(x_{j}+x_{k}\right) e^{(i \pi / 2 q)(2 q-1-k+j)} \\
& =(-1)^{r(l-r)} y_{\tilde{\rho} \tilde{\tilde{\sigma}}}^{-1} \exp \frac{i \pi}{2 q}\left[(l-r) \sum_{j \in \rho} j-r \sum_{k \in \tilde{\sigma}}(k+1)\right] .
\end{aligned}
$$

Empty products, which arise if $r=1$ or $l-1$, will be interpreted as equal to one. Multiplying the three partial products,

$$
m_{\rho \sigma}=(-1)^{r(l-r)}(2 i)^{u(l-1) / 2} S_{\rho} S_{\tilde{\sigma}} y_{\hat{\rho} \tilde{\sigma}}^{-1} \exp (i \pi / 2 q)(l-1)\left[\sum_{j \in \rho} j-\sum_{k \in \tilde{\sigma}}(k+1)\right] .
$$

The cofactor $M_{\rho \sigma}$ is by definition the signed minor of $\delta$ from the right, the rows indicated by $\tilde{\rho}$ in the top and by $\sigma$, in reverse order, in the bottom i.e. the rows omitted by $m_{\rho \sigma}$. The exponent of -1 in the sign, call it $s_{1}$, carried by $M_{\rho \sigma}$ is the sum of the row and column indices in $m_{\rho \sigma}$ (counting from 1 to $2 l$ ) and this sum is

$$
\sum_{j \in \mathcal{D}}(\hat{j}+1)+\sum_{k \in \tilde{\sigma}}(2 l-\hat{k})+l(l+1) / 2 .
$$

By (1) the unsigned minor $s_{1} M_{\rho \sigma}$ is equal to the factor $s_{2}=(-1)^{\Sigma_{j \in} j j+\Sigma_{k \in \sigma}(k+1)}$ times the left minor $m_{\tilde{\rho} \gamma}$ chosen from the same rows. 
The exponent of -1 in $s_{1} s_{2}$ is

$$
\begin{aligned}
\sum_{j \in \rho}(\hat{j}+1)+\sum_{j \in \tilde{\rho}} j+\sum_{k \in \sigma}(k+1)+ & \sum_{k \in \tilde{\sigma}}(2 l-\hat{k})+l(l+1) / 2 \\
& \equiv \sum_{j \in \rho} n_{j}+\sum_{k \in \sigma} n_{k}+\sum_{k \in L} n_{k}+l(l+1) / 2 \quad(\bmod 2) .
\end{aligned}
$$

Thus

$$
s_{1} s_{2}=(-1)^{N_{0}+N_{\sigma}+N+l(l+1) / 2}
$$

and

$$
\begin{aligned}
m_{\rho \sigma} M_{\rho \sigma} & =s_{1} s_{2} m_{\rho \sigma} m_{\tilde{\rho} \tilde{\sigma}} \\
& =c(-1)^{N_{\rho}+N_{\sigma}} S_{\rho} S_{\tilde{\rho}} S_{\sigma} S_{\tilde{\sigma}} y_{\rho \tilde{\sigma}}^{-1} y_{\sigma \tilde{\rho}}^{-1} \\
& =c(-1)^{N_{\rho}+N_{\sigma}} S_{\rho}^{\prime} S_{\tilde{\rho}} S_{\sigma}^{\prime} S_{\tilde{\sigma}} y_{\rho \sigma}^{2} \quad(\text { by (5)) } \\
& =m_{\sigma \rho} M_{\sigma \rho} .
\end{aligned}
$$

Equation (4) is verified.

If $\gamma=\left(\gamma_{0}, \gamma_{1}, \ldots, \gamma_{l-1}\right)$ is any vector of $l$ complex components let $\gamma^{\rho}=\prod_{j \in \rho} \gamma_{j}$, and $\bar{\gamma}^{\rho}=\prod_{j \in \rho} \bar{\gamma}_{j}$. Also, let $\hat{\beta}^{\rho}=\prod_{j \in \rho} \hat{\beta}_{j}$. In this notation

$$
\left|\begin{array}{ccc}
\beta_{j_{0}} \varepsilon_{0}^{j_{0}} & \cdots & \beta_{j_{0}} \varepsilon_{l-1}^{j_{0}} \\
\vdots & & \vdots \\
\beta_{j_{r-1}} \varepsilon_{0}^{j_{r-1}} & \cdots & \beta_{j_{r-1}} \varepsilon_{l-1}^{j_{r}-1} \\
\bar{\alpha}_{k_{l-r-1}} \varepsilon_{0}^{2 q-1-k_{l-r-1}} & \cdots & \bar{\alpha}_{k_{l-r-1}} \varepsilon_{l-1}^{2 q} \overline{1}^{1-k_{l-r-1}} \\
\vdots & & \vdots \\
\bar{\alpha}_{k_{0}} \varepsilon_{0}^{2 q-1-k_{0}} & \cdots & \bar{\alpha}_{k_{0}} \varepsilon_{l-1}^{2 q-1-k_{0}}
\end{array}\right|=\beta^{0} \bar{\alpha}^{\tilde{\sigma}} m_{\rho \sigma} .
$$

The cofactor of this term in the Laplace expansion of $D$ from the first $l$ columns is $\alpha^{\tilde{D}} \bar{\beta}^{\sigma} M_{\rho \sigma}$ (Figure 3).

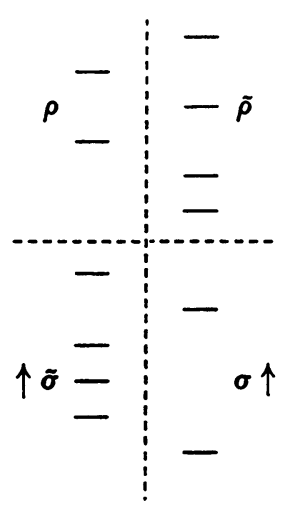

FIGURE 3 
Now $\beta^{\rho}=\prod_{j \in \rho} \beta_{j}=\prod_{j \in \rho}(-1)^{n_{j}} \hat{\beta}_{j}=(-1)^{N_{\rho}} \hat{\beta}^{\rho}$. Thus

$$
\beta^{\rho} \alpha^{\tilde{\rho}} \bar{\beta}^{\sigma} \bar{\alpha}^{\tilde{\sigma}} m_{\rho \sigma} M_{\rho \sigma}=\hat{\beta}^{\rho} \alpha^{\tilde{\alpha}} \hat{\beta}^{\sigma} \tilde{\alpha}^{\tilde{\sigma}}\left(c S_{\rho}^{\prime} S_{\tilde{\rho}} S_{\sigma}^{\prime} S_{\tilde{\sigma}} y_{\rho \sigma}^{2}\right)
$$

The sum

$$
\sum_{\rho, \sigma \in L_{r}} \beta^{\rho} \alpha^{\tilde{\rho} \bar{\beta}^{\sigma}} \bar{\alpha}^{\tilde{\sigma}} m_{\rho \sigma} M_{\rho \sigma}
$$

contains just those terms in the expansion of $D$ obtained by taking $r$ of the rows in the left minor from the top. It is a quadratic form $\left(A_{r} \gamma_{r}, \gamma_{r}\right)$ in the variables $\gamma_{r}=\left(\hat{\beta}^{\rho} \alpha^{\tilde{\rho}}\right)_{\rho \in L_{r}}$ with coefficient matrix $A_{r}=c\left(S_{\rho}^{\prime} S_{\tilde{\rho}} S_{\sigma}^{\prime} S_{\tilde{\sigma}} y_{\rho \sigma}^{2}\right)_{\rho, \sigma \in L_{r}}$. As remarked in the first paragraph of this section, $\left(y_{\rho \sigma}\right)_{\rho, \sigma \in L_{r}}$ is p.d. Since $S_{\rho}^{\prime}$ and $S_{\tilde{\rho}}$ are positive, $c^{-1} A_{r}$ is also p.d. This holds for $r=1,2, \ldots, l-1$.

Let $\gamma_{0}=\prod_{j \in L} \alpha_{j} \bar{\alpha}_{j}, \gamma_{l}=\prod_{j \in L} \beta_{j} \bar{\beta}_{j}=\prod_{j \in L} \hat{\beta}_{j} \overline{\hat{\beta}}_{j}$. The matrices $A_{0}=A_{l}$ accompanying $\gamma_{0}, \gamma_{l}$ in the Laplace expansion each have one element $d_{1} d_{4}=(-1)^{l} d_{2} d_{3}$. Since

$$
N=\sum_{j \in L} n_{j}=\sum_{j \in L} j+\sum_{k=0}^{l-1} k=G+l(l-1) / 2
$$

we conclude that $d_{1} d_{4}=c S^{2}$.

We have shown that

$$
D=\sum_{r=0}^{l}\left(A_{r} \gamma_{r}, \gamma_{r}\right)
$$

and that $c^{-1} A_{r}$ is p.d., $r=0,1, \ldots, l$. By hypothesis $\left|\alpha_{j}\right|+\left|\beta_{j}\right|>0, j \in L$. Hence there exists an $r, 0 \leqq r \leqq l$, and a $\rho \in L_{r}$ such that $\hat{\beta}^{\circ} \alpha^{\tilde{\rho}} \neq 0$. The vector $\gamma_{r}$ is not zero, and the lemma is established.

\begin{tabular}{|c|c|}
\hline$L$ & $D$ \\
\hline$\{1,2\}$ & $-e^{2 i \pi / 3}\left[\left|\beta_{1} \beta_{2}\right|^{2}+\left|\alpha_{1} \alpha_{2}\right|^{2}+\left|\beta_{1} \alpha_{2}\right|^{2}+\left|\beta_{2} \alpha_{1}\right|^{2}+2\left|\beta_{1} \alpha_{2}+\beta_{2} \alpha_{1}\right|^{2}\right]$ \\
\hline$\{0,2\}$ & $e^{2 i \pi / 3}\left[3\left(\left|\beta_{0} \beta_{2}\right|^{2}+\left|\alpha_{0} \alpha_{2}\right|^{2}+\left|\beta_{0} \alpha_{2}\right|^{2}+\left|\beta_{2} \alpha_{0}\right|^{2}\right)+\left|\beta_{0} \alpha_{2}-\beta_{2} \alpha_{0}\right|^{2}\right]$ \\
\hline$\{0,1\}$ & $-e^{2 i \pi / 3}\left[\left|\beta_{0} \beta_{1}\right|^{2}+\left|\alpha_{0} \alpha_{1}\right|^{2}+\left|\beta_{0} \alpha_{1}\right|^{2}+\left|\beta_{1} \alpha_{0}\right|^{2}+2\left|\beta_{0} \alpha_{1}+\beta_{1} \alpha_{0}\right|^{2}\right]$ \\
\hline$\{0,1,2\}$ & $\begin{array}{l}3\left(\left|\beta_{0} \beta_{1} \beta_{2}\right|^{2}+\left|\alpha_{0} \alpha_{1} \alpha_{2}\right|^{2}\right)+\left|\beta_{0} \alpha_{1} \alpha_{2}-\beta_{1} \alpha_{0} \alpha_{2}+\beta_{2} \alpha_{0} \alpha_{1}\right|^{2} \\
\quad+\left|\alpha_{0} \beta_{1} \beta_{2}-\alpha_{1} \beta_{0} \beta_{2}+\alpha_{2} \beta_{0} \beta_{1}\right|^{2} \\
\quad+\frac{2}{2}\left(\left|\beta_{0} \alpha_{1} \alpha_{2}-\beta_{2} \alpha_{0} \alpha_{1}\right|^{2}+\left|\alpha_{0} \beta_{1} \beta_{2}-\alpha_{2} \beta_{0} \beta_{1}\right|^{2}\right) \\
\quad+\frac{13}{2}\left(\left|\beta_{0} \alpha_{1} \alpha_{2}+2 \beta_{1} \alpha_{0} \alpha_{2}+\beta_{2} \alpha_{0} \alpha_{1}\right|^{2}+\left|\alpha_{0} \beta_{1} \beta_{2}+2 \alpha_{1} \beta_{0} \beta_{2}+\alpha_{2} \beta_{0} \beta_{1}\right|^{2}\right)\end{array}$ \\
\hline
\end{tabular}

It is possible to furnish a shorter proof if $l=q[13]$.

3. An illustration. In Table 1 we illustrate this lemma for the case $q=3$.

\section{TABLE 1}




\section{BIBLIOGRAPHY}

1. G. D. Birkhoff, Boundary value and expansion problems of ordinary linear differential equations, Trans. Amer. Math. Soc. 9 (1908), 373-395.

2. E. Coddington and N. Levinson, Theory of ordinary differential equations, McGraw-Hill, New York, 1955.

3. H. O. Cordes, Lecture notes on ordinary differential equations (Math. 219 B), Univ. of Calif., Berkeley, Calif., 1964.

4. I. Halperin, Closures and adjoints of linear differential operators, Ann. of Math. 38 (1937), 880-919.

5. E. Kamke, Differentialgleichungen, Chelsea, New York, 1948.

6. M. Marcus and H. Minc, A survey of matrix theory and matrix inequalities, Allyn and Bacon, Boston, Mass., 1964.

7. V. P. Mikhailov, On Riesz bases in $L^{2}(0,1)$, Dokl. Akad. Nauk SSSR 144 (1962), 981-984 = Soviet Math. Dokl. 3 (1962), 851-854.

8. Myravei, Trudy Mat. Inst. Steklov. (to appear).

9. B. Nagy, Spektraldarstellung linearer Transformationen des Hilbertschen Raumes, Springer, Berlin, 1942.

10. M. A. Naimark, Lineare Differentialoperatoren, Akademie-Verlag, Berlin, 1960.

11. G. Pólya and G. Szegö, Aufgaben und Lehrsätze aus der Analysis, Springer, Berlin, 1925.

12. M. L. Rasulov, Conditions for the normality of linear ordinary differential operators, Izv. Akad. Nauk Azerbaĭdžan. SSR Ser. Fiz-Mat. Tehn. Nauk 1953, no. 3, 110-118.

13. S. Salaff, A nonzero determinant related to Schur's matrix, Trans. Amer. Math. Soc. 127 (1967), 349-360.

14. M. H. Stone, A comparison of the series of Fourier and Birkhoff, Trans. Amer. Math. Soc. 28 (1926), 695-761.

\section{University of CALIFornia, Berkeley, California}

